\title{
La Comunicación política en los "Social media": Análisis comparado de la campaña de Barack Obama y Hillary Clinton en 2008.
}

\author{
María López-Trigo Reig \\ Universidad CEU Cardenal Herrera (Valencia) \\ maria.lopez3@uch.ceu.es \\ Rosario Garcia Cubells \\ Universidad CEU Cardenal Herrera (Valencia) \\ rgarcia@uch.ceu.es \\ Sandra Femenia Almerich \\ Universidad CEU Cardenal Herrera (Valencia) \\ sfemenia@uch.ceu.es
}

\section{Resumen}

El presente trabajo de investigación tiene por objeto el análisis de la comunicación on line del partido demócrata de los Estados Unidos en sus elecciones primarias del 2008. El interés del tema que se trata en esta investigación es el hecho que se observa, al estudiar la comunicación política de los últimos tiempos, un cambio del lenguaje debido a la revolución tecnológica, al desarrollo de nuevos canales y medios, y a la implicación de los ciudadanos en este desarrollo tecnológico.

Palabras clave: Marketing político, comunicación política, redes sociales, comportamiento político.

\section{The Political Communication in the "Social media": Comparative analysis of the campaign of Barack Obama and Hillary Clinton in 2008.}

\begin{abstract}
The present research aims to analyze online communication Democratic Party of the United States in 2008 primary elections. The interest of the topic covered in this research is the fact observed by studying political communication in recent times, a change of language because of the technological revolution, the development of new channels and media, and the involvement of the citizens of this technological development.
\end{abstract}

Key words: Political marketing, political communication, social networking, political behavior, comparative analysis.

\section{Referencia normalizada:}

López-Trigo Reig, M.; García Cubells, R. y Femenia Almerich, S. (2013) La comunicación política en los "Social Media". Análisis comparado de la campaña de Barack Obama y Hillary Clinton en 2008. Historia y Comunicación Social. Vol. 18. № Especial Diciembre. Págs. 813-826.

Sumario: 1.- Introducción, 2.- Metodología, 3.- Revisión Literaria, 4.- Resultados obtenidos, 5.Conclusiones, 6.- Referencias bibliográficas. 


\section{Introducción}

El desarrollo de la cultura digital como herramienta de comunicación entre la sociedad y el Estado es quizás la muestra principal del cambio de paradigma que está sufriendo en la actualidad la comunicación política (Rubio Núñez, R. 2007:193).

La irrupción de Internet supone un desafío para la política, un desafío para los medios de comunicación tradicionales- ellos también están en la red, y se enfrentan a fórmulas novedosas e imaginativas de comunicación, que ya no controlan -, y un desafío para la nueva ciudadanía de la democracia, cuya participación en el debate público pasa ahora por la red ( Rey Morato, J. Del 2008:303).

La comunicación política se va adaptando a esta revolución tecnológica y como se ve en el desarrollo de este estudio, son los nuevos canales de comunicación, la utilización de ellos por el ciudadano y la evolución transmedia los que van a marcar esta adaptación. No en vano son las nuevas tecnologías las que permiten la implicación del ciudadano en la comunicación política, de manera que, ahora, emisor y receptor se intercalan los papeles.

En este mismo sentido esta revolución de los medios debe servir para crear una mayor y mejor interacción de los políticos con los ciudadanos, la proximidad de los mensajes políticos en la Red tiene que servir para acercar a ambos colectivos, para escuchar al ciudadano y para comprender al político.

Maria José Canel le da mucha importancia a esta interacción definiendo comunicación política como "la actividad de determinadas personas (políticos, comunicadores, periodistas y ciudadanos) en la que, como resultado de la interacción, se produce un intercambio de mensajes con los que se articula la toma de decisiones políticas así como la aplicación de éstas en la comunidad" (Canel, M.J. 1999: 27).

En el año 2000 la campaña electoral norteamericana encontró definitivamente a Internet, y desde entonces irrumpen nuevas formas de comunicación para los candidatos hasta llegar al caso de estudio donde la utilización de la red ha sido protagonista indiscutible de esta campaña.

Para que los expertos en Marketing político sean conscientes de este cambio a la democracia participativa liderada por los nuevos medios, es importante que desarrollen lo que nos indica Marc Cortés de saber lo que "es estar "en red", donde las conversaciones entre todos los actores fluyen de forma pública y te exigen dejar de hacer un "marketing basado en ti" y hacer un "marketing basado en nosotros", basado en la figura de un consumidor colaborativo, participativo e interactivo", y en este análisis vamos ver qué importante fue el cambio de mentalidad y la interactividad, a través del medio on line, con el ciudadano de los EEUU (Cortés, M. 2008:6).

La comunicación política moderna ya no puede conformarse, como ocurría anteriormente, con la calidad literaria de los discursos políticos y con el brío de la retórica de quienes los pronuncian, máxime cuando el auge de las Nuevas Tecnologías de la información y Comunicación (New Technologies of Information and Communication 
NTIC) ha estado acompañado por una auténtica "globalización" de la información política mediatizada llegando socialización de la comunicaciónn más desarrollada.

Las campañas electorales del siglo XXI no podían permanecer ajenas al fenómeno: la red ha producido las primeras manifestaciones de la e-democracia. Cuando se habla de ciberdemocracia, democracia digital, democracia online, e-gobierno (e-goverment), democracia electrónica, (e-democraty), e-voto y e- ciudadanía, algunos episodios confirman que los candidatos tendrán que contar más con la red." (Rey Morato, J. Del 2008:303).

Hoy esta Oficina de Comunicación de la Casa Blanca cuenta con un equipo de "Nuevos Medios" desde donde se controla todas la comunicaciones on line. Barack Obama, el primer presidente estadounidense que ha hecho de la tecnología parte de su estilo de vida, incluido su inseparable Blackberry, es seguido todos los días por ocho millones de cibernautas en Facebook y 3.5 millones en Twitter, convirtiéndose así en la figura más vistas y leída cibernéticamente (Tobaccowala, R. 2008 http:// momentumradical.blogspot.com).

\section{Metodología}

\subsection{Objetivos de la investigación}

El objetivo de esta investigación es descubrir cómo el medio internet fue clave y decisivo en la evolucion de la comunicación política hacia interacción con el ciudadano y la participación del mismo en este desarrollo. El análisis de la comunicación on line del partido demócrata de los Estados Unidos en sus elecciones primarias del 2008. El interés del tema que se trata en esta investigación es el hecho que se observa, al estudiar la comunicación política de los últimos tiempos, un cambio del lenguaje debido a la revolución tecnológica, al desarrollo de nuevos canales y medios que evolucionan hacia la narrativa transmedia, donde la implicación de los ciudadanos en este desarrollo tecnológico es clave.

Para poder desarrollar el análisis comparado nos planteamos los siguientes objetivos de la investigación:

Primero: determinar las variables críticas de la comunicación on line de los dos candidatos: Barack Obama y Hillary Clinton. Donde podamos observar las diferencias de los resultados obtenidos en cada una de esas variables.

Segundo: identificar las diferencias cuantitativas en audiencia y cuota de las webs de ambos candidatos. Este análisis nos comparará los resultados que han alcanzado durante el periodo de análisis ambos candidatos.

Tercero: analizar los datos de Search Engine Optimization (SEO) (Optimización para Motores de Búsqueda) de ambos candidatos, que durante esos meses fueron 
objeto de búsqueda en el medio Internet y determinaremos el número de noticias relacionadas con esas tablas de datos, comparando los resultados obtenidos.

Cuarto: comparar el número de blogs que hablaban de ambos candidatos durante el periodo analizado. Pretendemos buscar en esta herramienta las diferencias de presencia de los candidatos.

Por último: determinar las diferencias en la utilización de las redes sociales por los dos candidatos. Describir las diferencias de actuación en las mismas, determinar el uso que cada candidato ha hecho del soporte social y calificar la interactuación con el ciudadano.

\subsection{Análisis comparado}

La metodología utilizada en este análisis es una metodología comparativa. Para analizar las variables de un fenómeno (campaña on line de Barack Obama) lo sometemos a comparación con otro (campaña on line de Hillary Clinton). Usando esta metodología se identifican las peculiaridades de un proceso comunicativo para someterlo a contraste con otro proceso. Además el objetivo no es otro que establecer identidades y diferencias entre ambos procesos para comprender la estrategia de comunicación en el medio Internet de Barack Obama en las elecciones primarias del Partido Demócrata del 2008.

La comparación es el método propio de las Ciencias Sociales, puesto que es el equivalente a la experimentación de las Ciencias Naturales (Duverger, M. 1971:411).

La investigación comparativa es estudiada por Leonardo Morlino quien destaca que los investigadores que se enfrentan a la investigación comparativa o a los estudios de caso afrontan un amplio abanico de cuestiones metodológicas, que están recibiendo una atención teórica y técnica creciente en Ciencia Política, en relación con el diseño, realización y presentación de su investigación. Iniciamos nuestro recorrido histórico, tal y como nos propone Leonardo Morlino, con Descartes y su Escuela de la Lógica: "la comparación se considera una confrontación entre un "más" y un "menos", entre "mejor" y "peor", una confrontación en la que los electos normativos del juicio desempeñan un papel muy importante".

\section{Revisión literaria}

El concepto campaña electoral se define como la fase específica y temporalmente delimitada durante la cual las fuerzas políticas concurrentes se presentan ante el electorado con sus programas, en competencia y discusión de los demás, interesando el apoyo de los de los ciudadanos. (Arnaldo y Delgado-Iribarren, 2009).

En este contexto, el marketing político es, según Ortega Martínez (2009), el marketing que pueden realizar las organizaciones políticas y poderes públicos, para 
influir en el comportamiento de los ciudadanos y alcanzar sus fines. De acuerdo con este concepto, dicho autor, apunta que el marketing político representa una parcela específica del marketing social, que en muchos casos pueden recibir el nombre de marketing de las instituciones o del sector público. También se percibe como marketing político no sólo la publicidad política, la comunicación política en las elecciones y los discursos electorales, sino que abarca el conjunto de herramientas del posicionamiento del partido en un mercado electoral. Introduciendo similitudes con el marketing comercial. (Harrop M. 1990:277). Por su parte, Kavanagh, D. (1996) delimita el marketing político electoral, como un conjunto de estrategias y herramientas para el seguimiento y estudio de opinión pública antes y durante una campaña electoral, para el desarrollo de campañas de comunicación y evaluar su impacto.

Para Maarek (2004) tres elementos principales explican la antelación del marketing político en Estados Unidos: el sistema electoral, la tradición de «comunicación democrática» de este país, y la anterioridad de la penetración de los modernos medios de comunicación de masas. Dentro de las peculiaridades del sistema electoral se describen las elecciones primarias, objeto de este trabajo. Así, el sistema de elecciones primarias implica, por su propia naturaleza, una doble incitación para desarrollar el «consumo» de comunicación política, cuantitativa, en primer lugar, porque el sistema de elecciones primarias duplica, de alguna manera, cada campaña electoral, que de esta forma está precedida de una campaña de primarias, en ocasiones hasta más larga. Así, las primarias presidenciales duran desde comienzos de enero hasta finales de junio, mientras que la campaña presidencial propiamente dicha, una vez han sido designados los candidatos de cada partido por su convención, empieza en julio y continúa hasta el primer martes de noviembre. Y cualitativa, en segundo término, puesto que, para ser designado candidato de su partido, un hombre político no sólo tiene que convencer a algunos caciques, sino que debe llevar a cabo una auténtica campaña entre los simpatizantes del partido, para convencerles de que le presten su apoyo en vez de hacerla con otro miembro de su propio partido, con ideas inevitablemente bastante próximas. Este tipo de campaña exige, en consecuencia, un esfuerzo de comunicación política paradójicamente más importante desde un punto de vista cualitativo, que el que exigirá luego la verdadera campaña: resulta más difícil, respecto del electorado, desmarcarse de un hombre de ideas afines que de un político de otro partido.

\section{4 . Resultados obtenidos}

El objeto de estudio es el análisis de la comunicación on line llevada a cabo en la campaña electoral de las elecciones primarias del Partido Demócrata de los Estados Unidos de 2008. En la que los dos candidatos, Barack Obama y Hillary Clinton se disputaban la candidatura de dicho partido a las elecciones presidenciales de ese mismo año. 
Se define el periodo de estudio de Enero a Mayo del 2008, ya que es el periodo el Partido Demócrata de los EEUU tiene determinado para sus convenciones internas y sus respectivas votaciones, en las que se elige el candidato a las elecciones presidenciales. El 3 de enero se celebra la primera convención en Iowa y el 3 junio finalizan en cuatro Estados.

Se describe el uso del medio Internet por ambos candidatos para enumerar y definir las variables estudiadas y de este modo pasar a describir el análisis comparado de la comunicación on line de la elecciones marco de este estudio. Si bien, el objeto de estudio de la investigación se centrará en un análisis de corte cuantitativo, como se muestra en las siguientes paginas, por lo que ahora en los siguientes parráfos sólo se ofrece un esbozo cualitativo de cómo se plantearon ambas campañas de comunicación política.

Al inicio del 2008 Obama era una especie de empresa pequeña sin marca, pero para principios de junio de ese mismo año, a solo 5 meses de la contienda, Obama se había convertido en una gran marca, gracias a su empuje, creatividad, talento e inteligencia, alzándose como la gran oportunidad histórica de esperanza y cambio que reclaman los norteamericanos, no contentos con las últimas actuaciones políticas de Bush.

El primer golpe de efecto de Barack Obama fue ganar Iowa. El triunfo de Obama no sólo responde a la publicación de sus dos libros biográficos, sino también a su gran capacidad e inteligencia organizativa, misma que adquirió desde los años ochentas como organizador de comunidades en Chicago. Ahí aprendió a trabajar por y con la gente en la lucha y la implementación estratégica, adquiriendo conocimientos y experiencias de contacto directo con los votantes, ya en aquel entonces con un visión de la utilización de los medios muy innovadora, mismas que le han sido sumamente útiles para ganar las elecciones primarias. Sus estudios en Ciencia Política en la Universidad de Columbia y de Derecho en Harvard, también le han sido, sin duda, de suma utilidad en su carrera rumbo a la Casa Blanca. (Renmick, D. 2010: 15).

En Iowa, utilizando su gran capacidad organizativa, dio un primer y sorpresivo "golpe" a sus adversarios, al ganar la contienda interna y poner a Hillary Clinton en un tercer lugar. En esta elección estatal, celebrada el 3 de enero del 2008, Obama logró alcanzar un 38 por ciento de los votos, venciendo con comodidad a John Edwards, que logró el 30 por ciento, y a Hillary Clinton, que logró el 29 por ciento. (www.realpolitik.com)

A partir de esta primera elección, en la que votaron 220.000 personas, Obama afinó su estrategia política, orientada a ganar las calles (a través del contacto directo de su estructura electoral con los ciudadanos) y a ganar los medios (el debate público), a través de una campaña electoral, tanto mediática en forma de spots publicitarios, como de presencia en las noticias y de entrevistas en los diferentes medios de comunicación y continuas acciones en el medio Internet, que le proporcionara un éxito en su comunicación. 
Desde los inicios de su campaña electoral, Obama acudió a una novedosa y astuta estrategia desde la red, utilizando las nuevas tecnologías de la información como parte de las herramientas no sólo para comunicarse y organizar a los votantes, sino también para recaudar los fondos que requería la misma campaña. De esta forma, se editó, por ejemplo, el video la Gran Chica Obama, mismo que circula por internet y ha alcanzado una gran popularidad.

Después del sorpresivo triunfo en Iowa y Carolina del Sur, Obama concentró sus esfuerzos en las elecciones del 12 de febrero del 2008, llamadas el "primer súper martes," día en el que una gran cantidad de estados (24) organizan sus elecciones primarias. A partir de esta fecha, lo que quedó claro para los norteamericanos es que la contienda definitiva al interior del Partido Demócrata sería entre Hillary Clinton y Barack Obama.

Para marzo de ese mismo año, no sólo aumentó Obama sus activos electorales y su presencia en los medios de comunicación, sino también la recaudación de fondos, ya que después del primer "súper martes" logró recaudar 7.2 millones de dólares que se sumaron a los 32 millones recaudados desde enero del 2008. Cuando llegó el segundo "súper martes," a inicios de marzo, Obama ya había afinado su estrategia centrada en la campaña mediática y de contacto directo, creando sendas estructuras para organizar y movilizar a los electores a lo largo y ancho de los Estados Unidos y aumentando su gasto en publicidad pagada en los medios de comunicación. Después llegó el "Momento Obama" en el cual el senador por Illinois se perfiló como el candidato adalid de la contienda, al ganar diez elecciones al hilo y posesionarse como el líder en las recaudaciones económicas, ya que para mayo del 2008 había recaudado más de 226 millones de dólares proveniente de más de 1.5 millones de personas. (Tobaccowala, R. 2008: http://momentumradical.blogspot.com)

Para principio de junio, después de las elecciones de Dakota del Sur y Montana y una vez que el Partido Demócrata había resuelto los polémicos casos de Michigan y Florida, Obama se proclamó como el vencedor al superar los 2,118 delegados que requería para ganar la nominación. La estrategia de los avales no sólo permitió darle una mayor relevancia mediática a la campaña de Obama, sino incluso sumar prestigios y abonar en la construcción del "nuevo mito de la política norteamericana," ya que a Obama se le compara mucho con el famoso ex presidente asesinado, John F. Kennedy, uno de los más queridos por los estadounidenses.

Una de las características distintivas de las primarias del Partido Demócrata, en el proceso de nominación de su candidato presidencial, fue el uso de las nuevas tecnologías de la información y las telecomunicaciones en la campaña. En lo particular, Obama sobresalió por el uso creativo e inteligente de Internet como instrumento para persuadir, organizar y movilizar a los electores, así como para recaudar fondos para el financiamiento de su campaña. De cierta manera, Obama hizo a través del uso de internet que su campaña fuera una campaña de la gente, una campaña transmedia. (Valdez Zepeda, A., Huerta Franco, D.A. 2008: www.revistalatinacs.org). 
Por el contrario la estrategia de Hillary estaba tan atrapada en los años noventa que cometió el tercer error fatal: Internet. No solo Hillary subestimó Internet como medio de comunicación, sino que jamás imaginó el poder de Internet para conseguir fondos de campaña e involucrar a la gente. Los Clinton diseñaron la mejor máquina de recolección de fondos del siglo 20, logrando que las personalidades del partido demócrata donaran la máxima cantidad posible para su causa, mientras que Obama diseñó la mejor máquina de recolección de fondos del siglo 21 (Pérez Carballada, C. 2008. www.marketisimo.com).

Más de 110 mil personas donaron fondos a la campaña de Barack Obama, lo cual le permitió afirmar: "hemos declarado la independencia de un sistema agotado, logrando la primera elección presidencial realmente financiada por el pueblo". Mientras Hillary Clinton se quedaba sin fondos, Obama batía todos los récords cuando sus seguidores donaban pequeñas sumas una y otra vez, y reforzaban su energía interactuando entre sí (Pérez Carballada, C. 2008: www.marketisimo.com).

Un grupo de asesores le enseñaron a usar la red 2.0 y armaron barackobama.com, la página de Internet que permitió armar una red social de apoyo a su causa, aplicando el efecto multiplicador de Internet a la tradicional red de voluntarios de una campaña política: más de 750 mil voluntarios se movilizaron fervientemente a través de Internet compartiendo fotos y vídeos, manteniendo chats sobre temas de interés y bajando ringtones con el eslogan "Si, podemos".

Se identifican las diferencias cuantitativas en audiencia y cuota de las webs de ambos candidatos, donde ya se pueden observar diferencias en los resultados. Datos conseguidos desde el consumo en el hogar y en el trabajo son superiores para Barack Obama en comparación con los conseguidos por Hillary Clinton. Durante cada uno de los meses analizados hay diferencia entre los datos obtenidos por cada uno de los candidatos, siendo Febrero el mes con resultados más altos. Por consiguientes se puede afirmar que hay diferencias cuantitativas en las variables analizadas.

Comparativa webs

\begin{tabular}{|l|c|c|}
\hline & \multicolumn{2}{c|}{ PROPIEDADES } \\
\hline \multicolumn{1}{|c|}{ UNIDADES } & Web B. Obama & Web H. Clinton \\
\hline Cuota \% hogar + trabajo & 1.96 & 1.17 \\
\hline Cuota \% hogar & 1.16 & 0.67 \\
\hline Audiencia hogar + trabajo (en miles) & 16,062 & 9,597 \\
\hline Audiencia hogar (en miles) & 8,795 & 5,093 \\
\hline
\end{tabular}

Fuente: Elaboración propia a partir de datos de Nielsen

Se analizan y comparan los datos resultantes de Seo de los dos candidatos, encontrando diferencia en los datos de cada uno de ellos. Detallando como palabras clave de búsqueda el nombre de pila de ambos candidatos en el través del buscador Google. Google trends nos muestra en los motores de búsqueda que ambos candidatos tienen 
una trayectoria similar a lo largo de los meses analizados, siempre teniendo resultados ligeramente superiores Barack Obama.

Comparativa SEO

\begin{tabular}{|c|c|c|}
\hline & \multicolumn{2}{|c|}{ PROPIEDADES } \\
\hline UNIDADES & B. Obama & H. Clinton \\
\hline Apariciones & 2.420 .000 & 1.039 .000 \\
\hline
\end{tabular}

Fuente: Elaboración propia a partir de datos de Google Trends

Se hace comparación del número de blogs que hablaban de ellos viendo en estos datos la diferencia de la presencia de cada uno de ellos en la red durante el periodo analizado. También se compara el número de noticias, de videos y de enlaces que citaban a los candidatos, determinando resultados muy diferentes para cada una de las variables.

Comparativa blogs

\begin{tabular}{|c|c|c|}
\hline & \multicolumn{2}{|c|}{ PROPIEDADES } \\
\hline UNIDADES & B. Obama & H. Clinton \\
\hline Apariciones & 14.300 .000 & 9.520 .000 \\
\hline
\end{tabular}

Fuente: Elaboración propia a partir de datos de Google Trends

Y por último se describen las diferencias en la utilización que de las redes sociales han hecho los dos candidatos, comparando los datos de Youtube donde los resultados dan ventaja en las tres variables a Barack Obama. El análisis de Facebook y en Twiter, donde los datos significativos a los que se tiene acceso hacen referencia a la usabilidad que le ha dado cada candidato a la herramienta y no tanto al dato cuantitativo que se ha conseguido a través de las mismas. En las dos redes sociales la interacción de Barack Obama con sus seguidores es superior a la que realiza su contrincante, consiguiendo de este modo tener más seguidos en las dos plataformas.

Las redes sociales fueron claves en el desarrollo de la narrativa transmedia, donde se observa una diferencia más amplia entre los dos candidatos. Por ello a continuación se hace un detalle de este comportamiento.

En Facebook, las notificaciones de ambos candidatos se suceden de una forma simultánea prácticamente todas el mismo día y con diferencia de horas entre ambos candidatos. 
Comparativa I uso facebook.

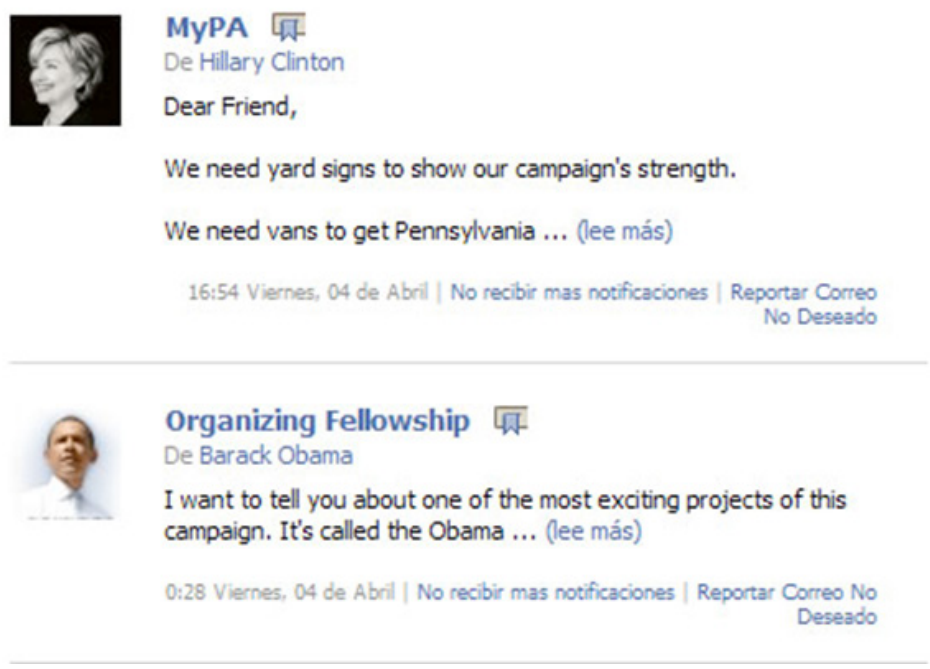

En cuanto a seguidores Obama gana por 782.789 partidarios frente a los 148.434 seguidores que obtiene Hillary a pesar de que Hillary presenta más comentarios en el Muro que el propio Obama. Pero la diferencia es que Hillary no desarrolla esos comentarios y se percibe como si estuviera telegrafiando sus mensajes, sin transmitir ninguna involucración ni interacción con el ciudadano. El lenguaje utilizado es diferente y por ello la respuesta es distinta.

La distribución de los perfiles es prácticamente similar: fotos, vídeos, noticias... pero la diferencia que más me ha llamado la atención es la siguiente:

Comparativa II uso facebook.

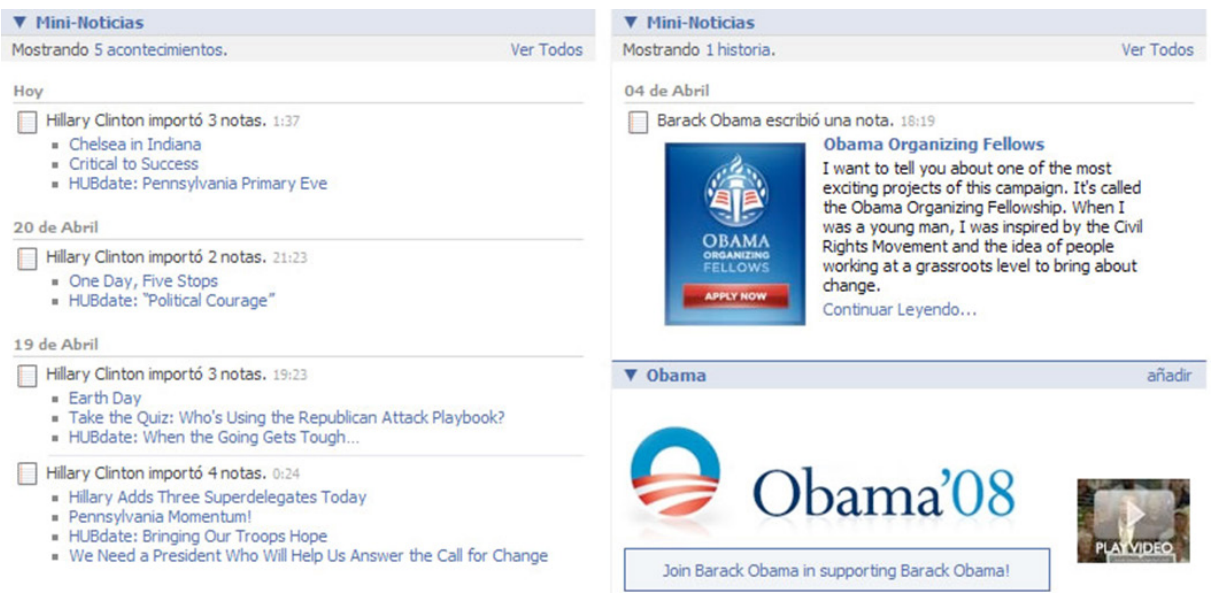


A la izquierda Hillary importando muchas notas a su perfil, a la derecha Obama escribiendo una nota. Hillary muestra titulares telegráficamente no necesariamente claros sobre su contenido sin embargo Obama nos cuenta, nos habla, y su comunicación es mucho más directa para los usuarios. Lo que Hillary importa como notas Barack las escribe directamente y eso se traduce también en una alta diferencia de participación por parte de los usuarios que opinan sobre la nota específica. Hillary informa, Obama comunica e implica. Hillary importa notas y fotos de otros medios o redes. Obama escribe notas y crea álbumes en Facebook. Utiliza realmente los servicios de la red social.

En el análisis de Twiter la diferencia es más evidente. Twitter es un medio en el que se dispone de muy pocos caracteres para comunicarnos y puede que para los políticos esto sea un handicap pero no ha de ser un impedimento.

Comparativa I Narrativa twiteer

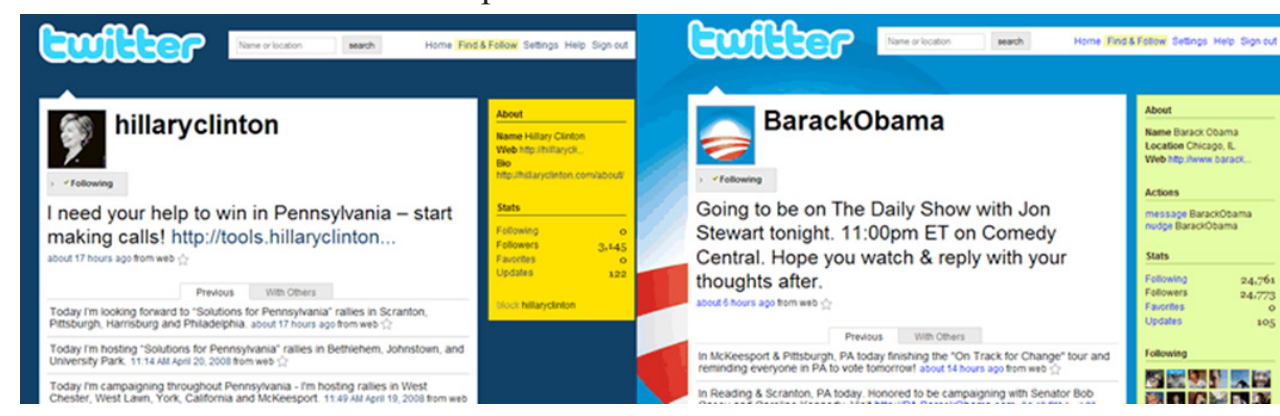

A la izquierda el perfil de Hillary Clinton mucho menos personalizado que el de Barack Obama. Pero ¿dónde está el secreto del éxito de Obama en Twitter? en ser bidireccional.

Comparativa II Narrativa twiter

\begin{tabular}{|c|c|c|c|}
\hline & & \multicolumn{2}{|l|}{ About } \\
\hline \multicolumn{2}{|l|}{ About } & \multirow{2}{*}{\multicolumn{2}{|c|}{$\begin{array}{l}\text { Name Barack Obama } \\
\text { Location Chicago, IL }\end{array}$}} \\
\hline \multirow{3}{*}{\multicolumn{2}{|c|}{$\begin{array}{l}\text { Name Hillary Clinton } \\
\text { Web http://hillarycli... } \\
\text { Bio } \\
\text { http://hillaryclinton.com/about/ }\end{array}$}} & & \\
\hline & & \multirow{2}{*}{\multicolumn{2}{|c|}{ Web http://www. barack... }} \\
\hline & & & \\
\hline \multicolumn{2}{|l|}{ Stats } & \multicolumn{2}{|c|}{$\begin{array}{l}\text { message BarackObama } \\
\text { nudge BarackObama }\end{array}$} \\
\hline \multirow{4}{*}{$\begin{array}{l}\text { Following } \\
\text { Followers } \\
\text { Favorites } \\
\text { Updates }\end{array}$} & o & & \\
\hline & 3,145 & \multicolumn{2}{|l|}{ Stats } \\
\hline & 0 & & \\
\hline & 122 & Following & 24,761 \\
\hline \multirow{3}{*}{\multicolumn{2}{|c|}{ block hillaryclinton }} & Followers & 24,773 \\
\hline & & Favorites & 0 \\
\hline & & Updates & 105 \\
\hline
\end{tabular}

Fuente : SearchMedia 
Hillary no sigue a nadie. Obama tiene casi tantos seguidores como usuarios a los que sigue.

\section{Conclusiones:}

Las características diferenciadas y el uso de los "social media" en la comunicación de Barack Obama le permitieron romper todos los moldes de lo establecido, se consiguió alcanzar cifras de audiencia, de seguimiento, de voluntarios, de presencia on line nunca vista antes en ninguna campaña de las elecciones primarias de los Estados Unidos. Fue el desarrollo de su comunicación on line una de las claves que le permitió pasar la contienda de las primarias y ser el primer candidato afroamericano a la presidencia de los EEUU.

Después de realizar todo este análisis comparado de la comunicación on line desarrollada de enero a mayo del 2008 por los dos candidatos demócratas, podemos verificar la hipótesis: La estructura de comunicación on line de Barack Obama presenta características diferenciadas a la desarrollada por Hillary Clinton.

A la luz de la verificación de la hipótesis principal de trabajo, se pueden realizar las siguientes consideraciones respecto de las subhipótesis:

Primera las webs oficiales de ambos candidatos son la plataforma enlace de toda su actuación en el medio on line, desde donde cada uno de ellos, lanza mensajes, convoca e interactúa. Pero cada uno desarrollando diferentes estrategias.

En segundo término los datos analizados en Google con referencia a la búsqueda de ambos candidatos nos reflejan una diferencia notable de la importancia que tuvo cada uno de ellos para el ciudadano estadounidense. Barack Obama consigue mejores resultados en todos los índices estudiados: número de noticias que hacían referencia a su campaña, número de blogs que dedicaban sus líneas a su estrategia, videos buscados, e incluso libros, su innovación en el uso los "social media" le ayudó en estos resultados.

En tercer lugar en el análisis de las redes sociales, teniendo carencia de datos cuantitativos, también podemos concluir que la estructura de comunicación utilizada por ambos candidatos presentar diferentes características.

Los jóvenes fueron esenciales para la utilización de Internet en la campaña, lo que permitió a Obama recibir más dinero de la gente que nadie en la historia y construir una base de millones de ciudadanos en Internet que luego se movilizaron en el puerta a puerta y en el teléfono. Mientras que el 59\% de los jóvenes usa Internet con fines políticos, tan sólo el $20 \%$ de los mayores de 65 años lo hace. Los jóvenes pro-Obama han utilizado masivamente sus redes, chats y foros en los espacios sociales de Internet, como Facebook o MySpace. Ello le ha permitido ser independiente de los grupos de presión, cuyas donaciones rechazó. Es más, la base de datos de sus donantes permite a la campaña de Obama enviar constante información, solicitar opinión y proponer 
actos de movilización al núcleo de apoyo, que se cuenta en millones de personas (Castells, M: 2008: http://webpolitica.blogspot.com).

La tecnología social ha ayudado al candidato a llegar a la presidencia. Como se han descrito hay claves de su estrategia on line que han permitido aventajar a Barack Obama frente a Hillary Clinton, dándole prioridad a la implicación de ciudadano y consiguiendo una recaudación record en la historia de la primarias de los EEUU.

Las características diferenciadas en la comunicación on line de Barack Obama le permitieron romper todos los moldes de lo establecido, se consiguió alcanzar cifras de audiencia, de seguimiento, de voluntarios, de presencia on line nunca vista antes en ninguna campaña de las elecciones primarias de los Estados Unidos. Fue el desarrollo de su comunicación on line una de las claves que le permitió pasar la contienda de las primarias y ser el primer candidato afroamericano a la presidencia de los EEUU.

\section{Referencias bibliográficas:}

ARNALDO, E. y DELGADO-IRIBAREN, M. (2009) "Diccionario electoral" Los consultores de los Ayuntamientos. La ley. Madrid.

CANEL, MJ (1999) Comunicación Política. Técnicas y estrategias para la sociedad de la información. Tecnos. Madrid.

CORTES, M., MARTINEZ PRIEGO, J.M., MORGE, S. (2009) Claves para entender el Nuevo marketing. Bubok Publishing. Madrid.

HARROP M. (1990) Asuntos parlamentarios. Macmillan. Londres.

KAVANAGH , D (1995) La campaña electoral: la nueva campaña de política. Lackwell publishers. Oxford.

MAAREK, P.J. (1995) Política de marketing y comunicación . John Libbey \& Co. Londres.

MAAREK, P.J. (2009) Marketing político y Comunicación. Claves para una nueva información política. Paidos. Barcelona.

MORLINO, L. (2010) Introducción a la Investigación Comparada. Alianza Editorial. Madrid.

ORTEGA MARTÍNEZ, E. (2009) El nuevo diccionario de marketing. Esic .Madrid. REMNINCK, D. (2010), El puente. Vida y Ascenso de Barack Obama. Debate, Barcelona.

REY MORATO, J.Del (2008) Comunicación política, Internet y Campañas electorales. De la teledemocracia a la ciberdemocracia. Tecnos. Madrid.

TOBACCOWALA，R. "Obama, un candidato digital” [en línea] en http://momentumradical.blogspot.com.5 junio 2008 (consulta 17 septiembre 2010).

VALDEZ ZEPEDA, A., HUERTA FRANCO, D.A. 2008: www.revistalatinacs. org). 
Páginas web

www.america.gov.

www.census.gov

www.democrats.org

www.eac.gov

www.edemocracia.com

www.marketisimo.org

www.revistalatinacs.org

\section{La autora}

María López Trigo Reig. Licenciada en Ciencias de la Información, especialidad de Publicidad y RRPP y Diplomada en Relaciones Pública, con más de 16 años de experiencia en diversas empresas y sectores de la Comunidad Valenciana. Estoy habituada a trabajar por objetivos y cumpliendo los presupuestos asignados a mi área de gestión. Me considero una persona con capacidades para la planificación, gestión y motivación de equipos. Soy una profesional entusiasta, dinámica y que lleva a cabo con éxito los retos que se propone. Actualidad: Vicedecana de Dirección de Empresas y Marketing. 